\title{
Tinea genitalis in a rural tertiary care hospital of Western U.P. India
}

\author{
Rameshwari Thakur ${ }^{1, *}$, Pragya Kushwaha², Avneet Singh Kalsi ${ }^{3}$, Paramjit Singh ${ }^{4}$ \\ ${ }^{1}$ Professor, ${ }^{2}$ Associate Professor, ${ }^{3}$ Medical Officer, ${ }^{4}$ Professor \& HOD, ${ }^{1,4}$ Dept. of Microbiology, ${ }^{2}$ Dept. of Dermatology, \\ Muzaffarnagar Medical College, Muzaffarnagar, Uttar Pradesh, India
}

*Corresponding Author:

Email: rameshwari_thakur@hotmail.com

\begin{abstract}
Introduction: Genital dermatophytosis is also known as tinea genitalis and pubogenital tinea, and it is one of the superficial fungal infections caused by dermatophytes. The classical clinical presentations in an immunocompetent person consist of an erythematous annular plaque with slightly raised scaly centrifugally advancing border and central clearing. The lesions in patients with HIV/AIDS or immunosuppressed individuals, can be extensive and without central clearing.

Purpose: The present study was conducted to know the current scenario and possible mode of transmission of tinea genitalis in males and females and to isolate the causative dermatophyte.

Materials and Methods: Patients with dermatophytic lesions in genital area and $\mathrm{KOH}$, and/ or culture positive were enrolled in the study. Samples were collected from the active borders of the lesions after cleaning it with $70 \%$ ethyl alcohol and were inoculated on Sabouraud Dextrose Agar (SDA), supplemented with chloramphenicol and cycloheximide. None of the patients had any immunosuppression except $2(0.72 \%)$ had diabetes mellitus and one $(0.36 \%)$ patient gave history of atopy.

Result: Out of a total 276 samples, 274 (99.27\%) were Trichophyton interdigitale, and two male patients (0.72\%) had tinea genitalis due to Trichophyton rubrum. History of use of topical steroid was present in $202(73.18 \%)$ patients.

Conclusion: Patients coming with tinea cruris should also be examined for the presence of tinea genitals, because according to our statistics $22.14 \%$ of them also had concomitant tinea genitals. History of having used topical corticosteroids should always be taken from them, because it results in extensive, atypical and extension of the lesions to the neighbouring anatomical sites.
\end{abstract}

Keywords: Tinea genitalis, Trichophyton interdigitale, Topical steroids.

\section{Introduction}

Dermatophytic infections are common superficial skin infection found all over the world and are otherwise called tinea or ringworm. ${ }^{1}$ Dermatophytes can be classified depending upon their natural habitat into anthropophilic, zoophilic and geophilic. Anthropophilic dermatophytes can cause human-to-human infection and is characterised by chronic and relatively low inflammatory response. Zoophilic dermatophytes cause animal infection, but can be transmitted to humans and are responsible for acute inflammatory lesions and are known to be highly contagious. Geophilic dermatophytes are found in soil and may cause sporadic human infection, which are inflammatory in nature.

The clinical diagnosis is given according to the anatomical site involved, e.g., when scalp, trunk and extremities, face, hand, groin, feet or nail are involved, it is called tinea capitis, tinea corporis, tinea faciei, tinea manuum, tinea cruris, tinea pedis and tinea unguium respectively. Among these, tinea corporis is the commonest clinical type, followed by tinea cruris.
Three genera of dermatophytes have been incriminated for causing superficial skin infection: Trichophyton, Microsporum and Epidermophyton. Sybren de Hoog et al., classifies dermatophytes into six biological classes based on multilocus genetic analysis, Trichophyton, Epidermophyton, Nannizzia, Microsporum, Lophophyton, and Arthroderma (unpublished data). ${ }^{2}$

Dermatophytes grow only within keratin layers and usually do not invade living epidermis. The predisposing factors include hot and humid, tropical climate, unhygienic conditions, atopy, diabetes, debilitating diseases including HIV infection. It is common in all age groups except tinea capitis, which occurs mostly in children.

India is a tropical country and due to its hot and humid climate, many people are affected. Patients with dermatophytic infection are seen throughout the year, but spikes are seen during rainy season. ${ }^{3}$ Several studies have been conducted in different parts of India; the most common dermatophyte incriminated is $T$. rubrum (Trichophyton rubrum). Some of the studies have been listed in the (Table1). ${ }^{4-18}$

Table 1: Results of various studies in India on prevalence of dermatophytes

\begin{tabular}{|l|l|l|l|}
\hline $\begin{array}{l}\text { Authors, year and } \\
\text { place of study }\end{array}$ & Predominant Species & $\begin{array}{l}\text { Second predominant } \\
\text { species }\end{array}$ & $\begin{array}{l}\text { Other } \\
\text { dermatophytes }\end{array}$ \\
\hline $\begin{array}{l}\text { Verma } \text { et } \text { al. } 2017^{4} \\
\text { Shimla, H.P. }\end{array}$ & $\begin{array}{l}\text { T. mentagrophytes } \\
62.28 \%\end{array}$ & T. rubrum $23.40 \%$ & $\begin{array}{l}\text { T. violaceum } 6.85 \% \\
\text { T. tonsurans } 2.85 \%\end{array}$ \\
\hline Zacharia et al. $2017^{5}$ & T. rubrum $41.2 \%$ & T. verrucosum $20.5 \%$ & T. tonsurans $14.7 \%$ \\
\hline
\end{tabular}




\begin{tabular}{|c|c|c|c|}
\hline Kerala & & & \\
\hline $\begin{array}{l}\text { Noronha et al. } 2016^{6} \\
\text { North Karnataka }\end{array}$ & $\begin{array}{l}\text { T. mentagrophytes } \\
48.3 \%\end{array}$ & T. rubrum $38.3 \%$ & T. violaceum 5\% \\
\hline $\begin{array}{l}\text { Choudhary \& Kumar. } \\
2016^{7} \\
\text { Bihar }\end{array}$ & T. rubrum $62.3 \%$ & $\begin{array}{l}\text { T. mentagrophytes } \\
14.1 \%\end{array}$ & E. flocossum $7.05 \%$ \\
\hline $\begin{array}{l}\text { Poluri LV. } 2015^{8} \\
\text { Telangana }\end{array}$ & T. rubrum $58.06 \%$ & $\begin{array}{l}\text { T. mentagrophytes } \\
22.58 \%\end{array}$ & \\
\hline $\begin{array}{l}\text { Lakshmanan et al. } 2015^{9} \\
\text { Tamil Nadu }\end{array}$ & T. rubrum $79 \%$ & $\begin{array}{l}\text { T. mentagrophytes } \\
14.5 \%\end{array}$ & \\
\hline $\begin{array}{l}\text { Surendran et al. } \\
2014^{10} \\
\text { Mangalore }\end{array}$ & T. rubrum $67.5 \%$ & $\begin{array}{l}\text { T. mentagrophytes } \\
20 \%\end{array}$ & \\
\hline $\begin{array}{l}\text { Bhatia VK, Sharma PC. } \\
2014^{11} \\
\text { Himachal Pradesh }\end{array}$ & $\begin{array}{l}\text { T. mentagrophytes } \\
63.5 \%\end{array}$ & T. rubrum $35.1 \%$ & $\begin{array}{l}\text { M. canis } 3.2 \% \\
\text { M. gypseum } 3.2 \%\end{array}$ \\
\hline $\begin{array}{l}\text { Pandey and Pandey. } \\
2013^{12} \\
\text { Gwalior, M P }\end{array}$ & T. rubrum $42.3 \%$ & $\begin{array}{l}\text { T. mentagrophytes } \\
12.7 \%\end{array}$ & \\
\hline $\begin{array}{l}\text { Sumana Khammam et } \\
\text { al. } 2004^{13} \\
\text { Andhara Pradesh }\end{array}$ & T. rubrum $60 \%$ & T. violaceum $26 \%$ & \\
\hline $\begin{array}{l}\text { Grover \& Roy. } 2003^{14} \\
\text { North-East India }\end{array}$ & T. tonsurans $20.5 \%$ & T. rubrum $9 \%$ & $\begin{array}{l}\text { M. feuugenium 5.8\% } \\
\text { T.mentagrophytes } \\
2.9 \%\end{array}$ \\
\hline $\begin{array}{l}\text { Bindu \& Pavithran. } \\
2002^{15} \\
\text { Calicut, Kerala }\end{array}$ & T. rubrum $66.2 \%$ & $\begin{array}{l}\text { T. mentagrophytes } \\
25 \%\end{array}$ & \\
\hline $\begin{array}{l}\text { Patwardhan et al. } 1999^{16} \\
\text { Aurangabad } \\
\text { Maharashtra }\end{array}$ & T. rubrum $28.12 \%$ & $\begin{array}{l}\text { T. mentagrophytes } \\
25.0 \%\end{array}$ & \\
\hline $\begin{array}{l}\text { Karmakar et al. } 1995^{17} \\
\text { Western Rajasthan }\end{array}$ & T. violaceum $55.76 \%$ & T. rubrum $42.3 \%$ & \\
\hline $\begin{array}{l}\text { Gupta et al. } 1993^{18} \\
\text { Ludhiana }\end{array}$ & T. rubrum $42.42 \%$ & E. flocossum $15.15 \%$ & $\begin{array}{l}\text { T. mentagrophytes } \\
6.06 \%\end{array}$ \\
\hline
\end{tabular}

Trichophyton interdigitale (T. interdigitale) formerly Trichophyton mentagrophytes, ${ }^{19}$ has been found to be the predominant dermatophyte species in our study. According to a recent study carried out by us at this tertiary care centre, Western U.P., India in 2014, T. interdigitale was the commonest dermatophyte species isolated $98.5 \%, T$. rubrum $0.5 \%$, and T. violaceum (Trichophyton violaceum) 1\% (unpublished data). T. violaceum mainly causes tinea capitis. We never got either a case of tinea cruris and tinea genitalis due to $T$. violaceum.

Some of the clinical conditions may mimic tinea genitalis e.g., candidiasis, psoriasis, eczema and erythrasma and for appropriate treatment, $\mathrm{KOH}$ mount and fungal culture should be performed. Tinea cruris presents as marked hyperpigmentation with active border showing erythematous papules, but these lesions become more extensive and atypical due to the misuse of topical steroid creams, which are easily available, overthe-counter in India.
Tinea genitalis once thought to be uncommon is now being reported with increased frequency. Verma and Vasani have recently published a comprehensive article on male genital dermatophytosis. ${ }^{20}$ They have mentioned in details the effect of unrestrained use of topical steroids. No study on female tinea genitalis has been reported from India so far. Keeping this in mind, we conducted a study on tinea genitalis in males and females and to find out the probable mode of transmission and also, to find out the dermatophytes species associated with it.

\section{Materials and Methods}

The study was conducted at a tertiary care centre of western U.P. in north India from July 2015 to June 2016. Inclusion criterion

1. Clinically typical or suspicious (atypical forms), but $\mathrm{KOH}$, and/or culture positive cases of tinea genitalis 
with or without tinea cruris between 15 to 55 years of age and of both sexes, were included in the study.

2. Data was collected in the form of age, sex, lesions occurring on penile shaft, preputial skin, glans and scrotum in males and on vulva, labia majora and labia minora with or without tinea cruris in females, were defined as tinea genitalis and were included in the study.

\section{Exclusion criterion}

1. Children below fifteen years and adults over 55 years of age.

2. Culture negative.

Data was collected regarding duration, foreign travel, pets, sports, and history of sexual contact, diabetes, atopy, history of presence of tinea cruris and tinea genitalis in spouse and treatment taken and history of use of topical and systemic steroids.

A total of 276 patients consisting of 174 males and 102 females were included in the study, which was conducted from July 2015 to June 2016. Patients were sent to microbiology laboratory for the collection and processing of the samples. The sample was collected from the suspicious lesions only after thoroughly cleansing the site with $70 \%$ ethyl alcohol. Those who had applied ointment, oil or cream, were instructed to take bath with soap and water and without applying anything, should come for the collection of the samples next day. The scales were collected using sterile scalpel blade from the active borders directly in a sterile petri dish. Collections of skin scrapings or nail clippings were done from other sites, if suspicious of dermatophytic infection and were processed separately. All the samples were subjected to potassium hydroxide $(\mathrm{KOH}) 20 \%$ mount and were examined under high power (40x) of the microscope to see the fungal elements, and then the samples whether $\mathrm{KOH}$ positive or negative, were inoculated on Sabouraud's Cycloheximide Chloramphenicol Agar (HiMedia). The petri plates were incubated for a period of four weeks at $25^{\circ} \mathrm{C}$ and were examined every week for the evidence of fungal growth. Colony characteristics were read for the culture positive plates both surface and obverse. Lacto phenol Cotton Blue (LCB) mounts by teased mount and scotch tape method were prepared to study the microscopic structures in detail. Some other tests like urease and in vitro hair perforation test were done for the differentiation of $T$. interdigitale from T. rubrum.

\section{Results}

All patients presented with non- inflammatory lesions and there was no involvement of lymph nodes. Most common age group of presentation in males was between 15-25 years, $96(55.17 \%)$, and in females between $26-35$ years, 48 (47.06\%) (Table 2).

Table 2: Age wise distribution of tinea genitalis

\begin{tabular}{|c|c|c|}
\hline Age & Male & Female \\
\hline $15-25$ & $96(55.17 \%)$ & $12(11.76 \%)$ \\
\hline $26-35$ & $36(20.69 \%)$ & $48(47.06 \%)$ \\
\hline $36-45$ & $18(10.34 \%)$ & $30(29.41 \%)$ \\
\hline $46-55$ & $24(13.79 \%)$ & $12(11.76 \%)$ \\
\hline Total & $174(100 \%)$ & $102(100 \%)$ \\
\hline $\begin{array}{l}\chi^{2} \text { (Chi square) value=59.301 at df 3 } \\
\text { p-value <0.0001 (Significant) }\end{array}$ \\
\hline
\end{tabular}

The most common mode of transmission in males was autoinoculation $78(44.83 \%)$ and in females was sexual transmission 48 (47.06\%) (Table 3) and (Fig. 3b, c, d and 4b). Topical steroid and systemic steroid abuse was clinically obvious in some patients such as annular scaly plaques with double edged border (Fig. 3a) and atrophy suggestive of use of topical steroid in a female patient, atrophic skin lesions in a 40-year-old female patient (Fig. 4c) and extensive scaly thick plaques over penile shaft and preputial skin due to intramuscular use of Triamcinolone acetonide (Fig. 1d).

Table 3: Possible mode of transmission of tinea genitalis

\begin{tabular}{|l|c|c|}
\hline \multicolumn{1}{|c|}{ Mode of transmission } & Male & Female \\
\hline Autoinoculation & $78(44.83 \%)$ & $30(29.41 \%)$ \\
\hline $\begin{array}{l}\text { Sharing clothes (bed linens, } \\
\text { towels, undergarments) }\end{array}$ & $32(18.39 \%)$ & $12(11.76 \%)$ \\
\hline $\begin{array}{l}\text { Sexual transmission } \\
\text { (commercial sex workers, } \\
\text { homosexuals, married } \\
\text { females from their spouses) }\end{array}$ & $18(10.34 \%)$ & $48(47.06 \%)$ \\
\hline $\begin{array}{l}\text { Others (poor personal } \\
\text { hygiene, hyperhidrosis, } \\
\text { tight fitting synthetic } \\
\text { garments) }\end{array}$ & $46(26.44 \%)$ & $12(11.76 \%)$ \\
\hline \multicolumn{2}{|c|}{ Total } & $174(100 \%)$ \\
\hline $\begin{array}{l}\chi^{2} \text { (Chi Square) value= 48.510 at df3 } \\
\text { p-value <0.0001 (Highly Significant) }\end{array}$ & $102(100 \%)$ \\
\hline
\end{tabular}


Out of a total 174 male patients, $171(98.27 \%)$ had tinea cruris (Fig. 1a, b, c, d) and (Figure 2 a, b) and 9 (5.17\%) had concomitant tinea corporis (Fig. 1d).

Rest $3(1.72 \%)$ patients presented with solitary lesions involving genitalia (Fig. 2c, d).

Among 102 female patients, 99 (97.05\%) had tinea cruris (Fig. 3a, 4a,c, d) and $12(11.76 \%$ ) had concomitant tinea corporis (Fig. 4a). Only $3(2.94 \%)$ female patients had tinea genitalis, without tinea cruris or dermatophytic infection of any other body site (Fig. 4b).

History of having used topical steroid was present in $202(73.18 \%)$ patients (Fig. 5 a). Clinically, $238(86.23 \%)$ patients had lesions suggestive of having had topical steroid cream.

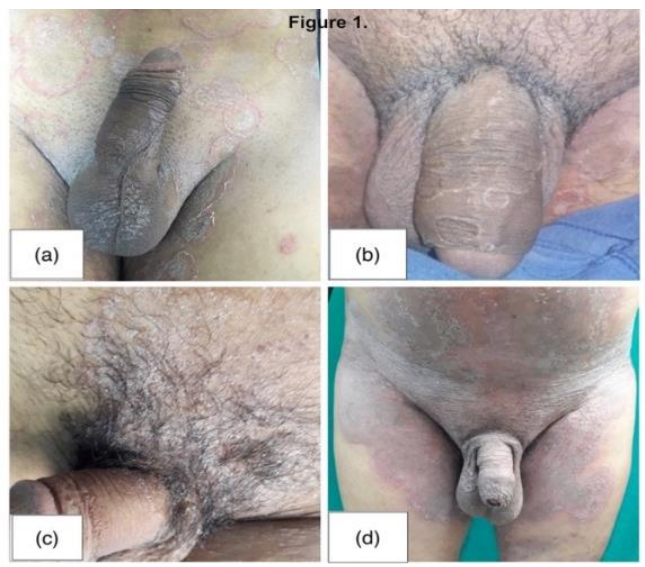

Fig. 1: Tinea genitalis in males: (a) Tinea genitalis involving scrotum, penis (dorsal and ventral aspects) and preputial skin with typical scaly annular lesions with central clearing in the groin; (b) Multiple annular scaly plaques over dorsal and ventral surfaces of penile shaft and scrotum; (c) Diffuse annular scaly plaque present in pubic region without central clearing and extending over scrotum and dorsal surface of penile shaft in a 29 years old male; (d) Extensive scaly thick plaques over abdomen, groin and penile shaft along with involvement of preputial skin and scrotum. This 40 year old patient was prescribed intramuscular injections of Triamcinolone acetonide and salicylic acid for topical application by a general practitioner

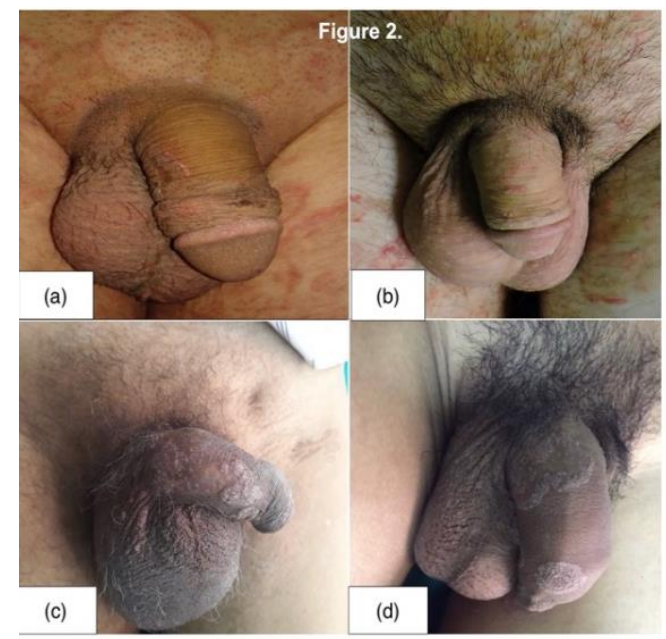

Fig. 2: Tinea genitalis in males: (a) Tinea genitalis presenting with ill-defined erythematous lesions over penile shaft and preputial skin. Erythematous annular lesions with central clearing in groin; (b) Tinea genitalis with erythematous and partially annular lesions in groin, penile shaft and glans penis; (c) Isolated scaly annular plaques on penile shaft (dorsal and ventral aspects) with papular lesions on scrotum. No history of sexual contact and of topical steroid use; (d) Isolated scaly plaques over the penile shaft and involvement of scrotum and no lesions in groin

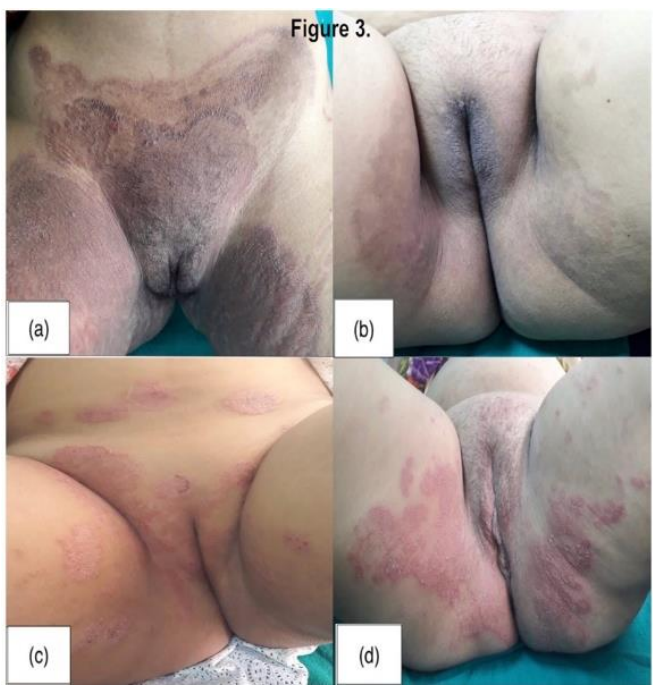

Fig. 3: Tinea genitalis in females: (a) Annular scaly plaques with double-edge, and atrophy of skin with history of use of topical steroids; (b) Healed hyper pigmented lesions. Patient got infection from her spouse; (c)24 years old female with annular erythematous scaly lesions on genitalia, also scattered on abdomen, got infection from her spouse; (d) Erythematous scaly plaques first appeared in medial sides of both thighs and buttocks and then spread to labia majora and mons pubis. Got infection from her partner 


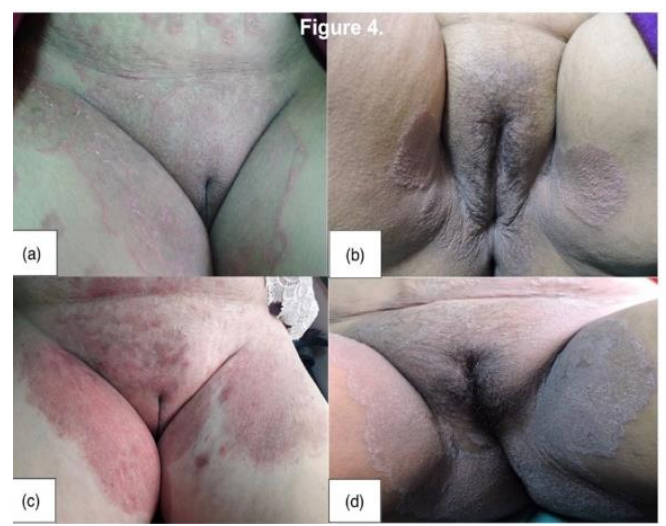

Fig. 4: Tinea genitalis in females: (a) Tinea genitalis and tinea cruris et corporis, annular erythematous scaly lesions with central clearing; (b) Tinea genitalis with bilateral annular plaques and involvement of labia majora without central clearing; (c) Erythematous scaly plaques over lower abdomen, mons pubis, thighs, groin and labia majora, atrophic skin at some places showing the long-term use of topical steroids; (d) Well-defined scaly plaques over mons pubis, labia majora, both thighs and groin

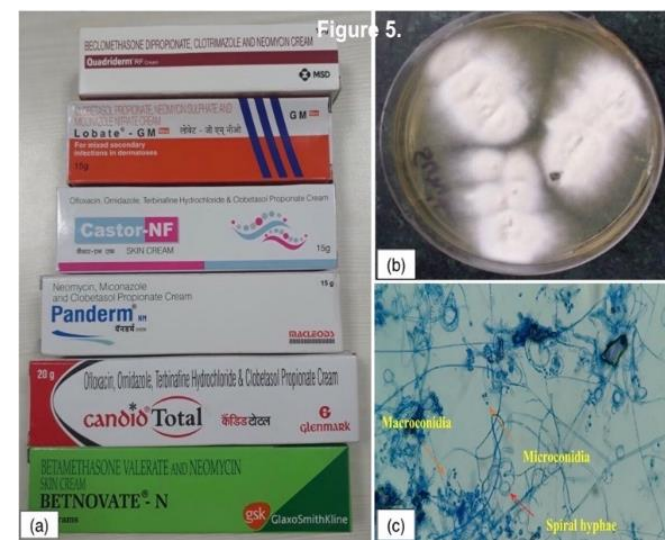

Fig. 5: (a) Various combinations of topical steroids, antifungal and antibacterial creams in irrational combinations sold as over- the-counter (OTC) creams; (b) Cottony beige coloured growth of $T$. interdigitale after one week incubation on SDA at $25^{\circ} \mathrm{C}$; (c) Lacto Phenol Cotton Blue (LPCB) mount

A total of 271(98.188\%) patients with tinea genitalis were $\mathrm{KOH}$ positive and 276 were culture positive. 274 (99.27\%) cultures grew T. interdigitale and $2(0.72 \%) T$. rubrum.

Colonies of T. interdigitale were powdery to fluffy, cream to white on obverse and yellow to brown on reverse (Fig. 5 b). Lactophenol cotton blue mounts were prepared, which showed septate fungal hyphae, with numerous spherical microconidia arranged in grape-like clusters, cigar-shaped macroconidia and spiral hyphae (Fig. 5 c).

T. interdigitale was isolated from 274 patients and $T$. rubrum was isolated from $2(0.724 \%)$.

\section{Discussion}

Hot and humid climate of western Uttar Pradesh (U.P.), low socio-economic status, along with misuse of topical steroids are possibly responsible for such a large number of dermatophytic infections including tinea genitalis. One third of daily OPD (Out-patient department) attendance consists of patients with various dermatophytic infections. Extensive and atypical tinea corporis with no central clearing is the most common clinical presentation, followed by tinea cruris, tinea genitalis, tinea capitis, tinea pedis, and tinea unguium, in descending order.

Although, there are several modes of transmission of tinea genitalis (Table 3), but the extension of the preexisting lesion of tinea cruris seems to be the one of the common reasons and especially after the use of topical steroid cream. ${ }^{20}$ The indiscriminate use of topical steroid is rampant in our country and these are easily available as over-the-counter drug due to laxity of drug regulatory authorities in India.

The diagnosis of tinea genitalis can be easily missed if the patient is not examined properly. According to Hebra (1860), 'When a patient thus affected lies with both thighs abducted and flexed, the whole extent of the disease is visible at a glance and it is then seen how remarkably exempt the penis and scrotum remain' ${ }^{21}$

Though some of the studies outside India have mentioned association of tinea genitalis due to autoinoculation from tinea pedis and onychomycosis of toenails especially when it was due to $T$. rubrum. Romano et al., (2007), ${ }^{22}$ and Ginter-Hanselmayer et al., (2016) have mentioned association of tinea genitalis due to autoinoculation from tinea pedis and onychomycosis of toenails. ${ }^{23}$ Surprisingly, none of the patients with tinea cruris or tinea genitalis had either involvement of the feet or toe nails due to tinea infection. People in developed nations often wear shoes, which lead to sweating and thus create good environment for the dermatophyte to grow. ${ }^{24}$ In India, most of the people wear open shoes, and so less chances for the development of onychomycosis.

Some of the female patients gave history of acquiring tinea genitals soon after marriage from their spouses (connubial) and they presented with initial lesions in the genital area and then spread to involve groin (Fig. 3c \& 4b). Mølenberg (2009) has reported connubial tinea gladiatorum due to T. mentagrophytes. ${ }^{25}$ Also, some researchers have mentioned infections transmitted by sexual contact in partners recently. ${ }^{23,26,27}$

Penile infections due to dermatophyte has been mentioned in literature due to wearing of tight loin cloth and if due to $T$. mentagrophytes, may rapidly involve the chest, back, legs, and feet and cause a severe, incapacitating inflammatory disease.$^{28}$ Even in extensive tinea cruris, the scrotum and penis are clinically unaffected. Involvement of glans penis is considered even rarer. ${ }^{29}$ The low incidence of scrotal dermatophytosis, even in the presence of extensive involvement of groin and thighs, has been ascribed to 
capric acid, one of the fatty acids in the epidermal barrier, particularly abundant in scrotal skin, thought to act as antifungal factor. ${ }^{30}$ According to Pillai et al., (1975), the rarity of infection of penile shaft could be due to decreased eccrine sweat secretions resulting in decreased hydration of the penile shaft. ${ }^{31}$ La Touche (1967) has found that in many cases of tinea cruris, the scrotum is culturally positive, even in the absence of clinical signs. It could be possible that scrotum may act as a reservoir for recurrent infections. ${ }^{32}$ Also, Hopkins et al., (1947) reported that one-eighth to one-third of military recruits with tinea cruris had positive fungal culture of the scrotum. ${ }^{33}$

Presence of tinea cruris along with tinea genitalis varies in different studies. In a study in Italy by Romano et al., (2007), genital infections accounted for $2 \%$ of cases of tinea cruris. ${ }^{22}$ Pandey et al., (1981), on the other hand found $20 \%$ of genital involvement among patients with tinea cruris. ${ }^{34}$ According to our statistics, $22.14 \%$ of patients with tinea cruris had concomitant tinea genitalis. It has been reported that genital dermatophytosis is more often seen in tropical countries, where hot and humid climate leads to local humidity and skin maceration. ${ }^{35,36}$ Moreover, friction involved during sexual intercourse can accelerate direct transmission, which is supported by a case series study of Otero et al., (2002), ${ }^{37}$ and Bakare et al., (2002) describing prostitutes in Spain and Namibia suffering from tinea cruris [38]. There are many studies, where the female partners were not affected. ${ }^{20,39,40}$

Extensive studies carried out recently by Verma and Madhu (2017) and Panda and Verma (2017), who also have great expertise in dermatophytosis, have mentioned about the inadvertent and unethical use of topical steroids, due to which dermatophytosis has acquired epidemic proportion and also tinea genitalis is being reported with increased frequency. ${ }^{41,42}$ The scenario here in Western U.P., is very similar to their description except in this region, $T$. interdigitale (phenotypically and clinically appears anthropophilic) predominates. Onethird cases in the dermatology outpatient department come with extensive dermatophytosis.

A recent article on tinea genitalis from Switzerland by Luchsinger et al., (2015), have mentioned seven tourists including males and females developed tinea genitalis on their visit to south East Asia. They had sex with commercial sex workers and got infected with $T$. interdigitale (zoophilic strain). On return to their country, they immediately went for medical advice due to acute symptoms and had inflammatory genital lesions. ${ }^{26}$ In India, most of the cases of tinea genitalis reported until now are due to anthropophilic species e.g., T. rubrum, T. interdigitale and E. flocossum, ${ }^{19,20,35,36}$ but tinea genitalis due to zoonotic species, e.g., Microsporum canis has been reported from Austria, Bosnia and Herzegovina and Italy, ${ }^{23,40,44} T$. interdigitale (zoonotic strain) from Thailand, ${ }^{26}$ and Trichophyton species of Arthroderma benhamiae has been reported from Germany. ${ }^{23}$ Human to human transmission has been found in $T$. interdigitale (zoonotic strain). ${ }^{26,27}$

So, tinea genitalis can be associated with tinea cruris, it can be either due to autoinoculation from tinea pedis or toenail onychomycosis. ${ }^{22,23}$ The lesions can be isolated involving the genitalia without dermatophytic infection either of crural region or of any other anatomical site. ${ }^{39,40,43-46}$

Diagnosis of the causative dermatophyte is important in order to treat and control the infection. Fungal culture should be done after clinical cure following the course of the antifungal treatment to confirm mycological cure. Treatment is not always easy, especially when the patients have used topical steroid. Longer period of antifungal treatment with higher doses are required and should be tapered gradually. ${ }^{47}$ Most of the studies in India show the predominance of T. rubrum and so the causative fungus commonly found in India is $T$. rubrum and next in frequency are $T$. interdigitale and E. floccousum. M. canis is the predominant cause of tinea genitalis in some of the European countries. ${ }^{23,40}$ Recently, Trichophyton species of Arthroderma benhamiae (zoophilic dermatophyte) has been incriminated as a cause of tinea genitalis. ${ }^{23,48}$ Usually, tinea genitalis is a superficial infection, but genital shaving can predispose the person to the development of deeper infection due to the involvement of the hair follicle leading to the formation of Majocchi's granuloma. Such conditions have ben reported due to infection with zoophilic dermatophytes. ${ }^{27,48}$

All the isolates of $T$. interdigitale tinea genitalis phenotypically appeared like anthropophilic strains and clinically also the lesions looked like due to anthropophilic strain. They were not inflammatory or ulcerative like in case of zoophilic infections. The anthropophilic species usually cause more chronic, less circumscribed infections, which result in less resistance to re-infection.

The colonies of anthropophilic strains are cottony in appearance and that of zoophilic strains have granular appearance. At times, the colony may have mixed appearance.

Limitation of the study is that we did not do the molecular typing of the isolates. It is usually not possible to differentiate between the anthropophilic strains and zoophilic strains of $T$. interdigitale on morphological and biochemical basis alone. Clinically it is seen that infection due to zoophilic strains is acute and inflammatory and at times, may heal with scarring. Infection due to anthropophilic strain is chronic and contagious. But, molecular identification methods are needed for the differentiation of zoophilic strains of $T$. interdigitale, T. mentagrophytes, and Trichophyton anamorph of Arthroderma benhamiae. ${ }^{19}$

\section{Conclusion}

Promiscuous society, tight fitting synthetic garments, easy access to the over the counter drugs like 
potent topical steroid creams unethically mixed with antifungal and antibiotic creams, are the possible contributing factors in rising cases of tinea genitalis.

Inadvertent use of topical steroids and relative lack of local cutaneous immunity are probable factors for persistent nature of the disease, results in extensive and atypical lesions and delay the appropriate treatment. Also, antifungal resistance, biofilm formation by the fungus as virulence factor and clinical resistance are serious challenges and patient should be treated suitably.

Funding: No funding sources.

Conflict of interest: None declared.

\section{References}

1. Vena, G.A., Chieco, P., Posa, F., Garofalo, A., Bosco, A., Cassano, N. Epidemiology of dermatophytosis: retrospective analysis from 2005 to 2010 and comparison with previous data from 1975. New Microbiol 2012;35(2):207-13.

2. Zhan, P., Liu, W. The Changing Face of Dermatophytic Infections Worldwide. Mycopathol 2017;182:77-86. DOI: org/10.1007/s11046-016-0082-8

3. Vora, N.S., Mukhopadhyay, A.K. Incidence of dermatophytosis of penis and scrotum. Indian J Dermatol Venereol Leprol 1994;60:89-91.

4. Verma, S., Verma., G, Sharma., V, Bhagra, S., Negi, A., Tegta, G.R. Current Spectrum of Dermatophytosis in a Tertiary Care Hospital of North India-A six year Clinicomycological Study. J Med Sci Clin Res 2017;5(3):1948894.

5. Zacharia, M., Kunjukunju, B.P. Clinical profile of patients with chronic dermatophytosis: a descriptive study from a tertiary care centres in Kerala. J Evid Based Med Health Care 2017;4(47):2863-66.

6. Noronha, T.M., Tophakhane, R.S., Nadiger, S. Clinicomycological study of dermatomycoses in a tertiary-care hospital in North Karnataka. Indian Dermatol Online J 2016;7:264-71.

7. Chaudhary, J.K., Kumar, A. A Clinico-Mycological Profile of Dermatophytosis at a Tertiary Care Hospital in Bihar. Int J Curr Microbiol App Sci 2016;5(2):181-89.

8. Poluri, L.V., Indugula, J.P., Kondapaneni, S.L. Clinicomycological study of dermatophytosis in South India. J Lab Phys 2015;7:84-9.

9. Lakshmanan, A., Ganeshkumar, P., Mohan, S.R., Hemamalini, M., Madhavan, R. Epidemiological and clinical pattern of dermatomycoses in rural India. Indian J Med Microbiol 2015;33:S134-S136.

10. Surendran, K., Bhat, R.M., Boloor, R., Nandkishore, B., Sukumar, D. A Clinical and mycological Study on Dermatophyte Infections. Indian J Dermatol 2014;59(3):262-67.

11. Bhatia, V. K., Sharma, V.K. Epidemiological studies on Dermatophytosis in human patients in Himachal Pradesh, India. Springer Plus 2014;3:134-40.

12. Pandey, A. Pandey, M. Isolation and Characterization of dermatophytes with tinea Infection at Gwalior (M.P.) India. Int J Pharma Sci Invent 2013;2:5-8.

13. Sumana, V., Singaracharya, M.A. Dermatophytosis in Khammam (Khammam district, Andhra Pradesh, India). Indian J Pathol Microbiol 2004;47(2):287-9.

14. Grover, S., Roy, P. Clinico-mycological Profile of Superficial Mycosis in Northeast India. MJAFI 2003;59(2):114-16.
15. Bindu, V., Pavithran, K. Clinico - mycological study of dermatophytosis in Calicut. Indian J Dermatol Venereol Leprol 2002;68:259-61.

16. Patwardhan, N., Dave, R. Dermatomycoses in and around Aurangabad. Indian J Pathol Microbiol 1999;(4):455-62.

17. Karmarkar, S., Kalla, G., Joshi, K.R., Karmarkar, S. Dermatophytoses in a desert district of Western Rajasthan. Indian J Venereol Leprol 1955;61:280-83.

18. Gupta, B.K.; Kumar, S.; Kumar, R.A.; Khurana, S. Mycological aspects of dermatomycoses in Ludhiana. Indian J Pathol Microbiol 1991;36(3):233-37.

19. Nenoff, P., Herrmann, J., Gräser, Y. Trichophyton mentagrophtes sive interdigitale? A dermatophyte in the course of time. Dtsch Dermatol Ges 2007;5:198-202. DOI: 10.1111/j.1610-0387.2007.06180.x

20. Verma, S.B., Vasani, R. Male genital dermatophytosis Clinical features and the effects of the misuse of topical steroid combinations-An alarming problem in India. Mycoses 2016;59:606-14. DOI: 10.1111/myc.12503

21. Hebra, F. Handbuch der speciellen Pathologic and Therapie (Virchow R, Erlagen Ed, Enke III, 1860-31. (Translated by) Hilton-Fagge and Pye-smith P.H. in 'On Disorders of skin', London, New-Syndenham Society', I La Touche C. J. Scrotal dermatophytosis: an insufficient documented aspect of Tinea cruris. Br J Dermatol 1967;79: 339-44.

22. Romano, C., Ghilardi, A., Papini, M. Nine male cases of tinea genitalis. Mycoses 2005;48:202-4. DOI: 10.1111/j.1439-0507.2005.01127.x

23. Ginter-Hanselmayer, G., Nenoff, P., Kurrat, W., Propst E., Durrant-Finn et al. Tinea in genital area. Der Hautarzt 2016;67(9):688-99. DOI: 10.1007/s00105-016-3848-5

24. Aly, R. Ecology and epidemiology of dermatophyte infections. J Am Acad Dermatol 1994;31:21-5. DOI: org/10.1016/S0190-9622 (08) 81262-5

25. Mølenberg, D., Deleuran, M., Sommerlund, M. Connubial tinea gladiatorum due to Trichophyton mentagrophytes. Mycoses 2010;53:533-34. DOI: 10.1111/j.1439-0507.2009.01734.x

26. Luchsinger, I., Bosshard, P.P., Kasper, R.S., Reinhardt, D., Lautenschlager, S. Tinea genitalis: a new entity of sexuality transmitted infection? Case series and review of literature. Sex Transm Infect 2015;1-4. DOI: 101136/sextrans-2015-052036

27. Galo, J.G., Woods, M., Grahm, R.M. Jennison., A.V. A severe transmissible Majocchi's Granuloma in an immunocompetent returned traveller. Med Mycol Case Rep 2017;18:5-7. DOI: org/10.1016/j.mmcr.2017.07.003

28. Rippon, J.W. Dermatophytosis and Dermatomycosis. In: Medical Mycology: The Pathogenic Fungi and Pathogenic Actinomycetes. 3rd ed. Philadelphia, PA: Saunders $1988 ; 169-175$.

29. D'Antuono, A., Bardazzi, F., Andalou. F. Unusual manifestations of dermatophytosis. Int J Dermatol 2001;40:164-66. DOI: org/10.1046/j.13654362.2001.00154.X.

30. Smith, J.G., Fisher, R.W., Blank, H. The epidermal barrier: a comparison between scrotal and abdominal skin. J Invest Dermatol 1961;36:337-41.

31. Pillai, K.G., Singh, G., Sharma, B. M. Trichophyton rubrum infection of the penis. Dermatologica 1975; 150:252-54.

32. La Touche, C. J. Scrotal Dermatophytosis. An insufficiently documented aspect of tinea cruris. $\mathrm{Br} \mathrm{J}$ Dermatol 1967;79:339-44. DOI:org/10.1111/j.13652133.1967.tb1504.x

33. Hopkins, J.G., Hillegas, A.B., Ledin, R. B., Rebell, G.C., Camp, E. Dermatophytosis in an infantry post: incidence 
and characteristics of infection by three species of fungi. J Invest Dermatol 1947;8:291-316.

34. Pandey, S.S.; Chandra, S.; Guha, P.K.; Kaur, P.; Singh, G. Dermatophyte infection of penis: associated with a particular undergarment. Int J Dermatol 1981;20:112-14.

35. Mukhopadhyay, A.K. Trichophyton rubrum infection of prepuce. Indian J Dermatol Venereol Leprol 2005;71:130-31.

36. Kumar, B., Talwar, P., Kaur, S. Penile tinea. Mycopathol 1981;75:169-77.

37. Otero, L., Palacio, V., Vazquez, F. Tinea cruris in female prostitutes. Mycopathol 2002;153:29-31.

38. Bakare, R.A. Oni, A.A., Umar, U.S., Adewole, I.F. et al. Pattern of sexually transmitted diseases among commercial sex workers (CSWs) in Ibadan, Nigeria. Afr J Med Sci 2002;31:243-47.

39. Das, J.K., Sengupta, S., Gangopadhyay, A. Dermatophyte infection of the male genitalia. Indian J Dermatol 2009;54:21-3.

40. Prohic, A.; Krupalija-Fazlic, M.; Sadikovic, T.J. Incidence and etiological agents of genital dermatophytosis in males. Med Glas (Zenica) 2015;12:52-6.

41. Verma, S.B., Madhu, R. The great Indian Epidemic of superficial dermatophytosis: An appraisal. Indian $J$ Dermatol 2017;62:227-36. DOI:10.4103/ijd.IJD_206_17

42. Panda, S.; Verma, S. The menace of dermatophytosis in India: The evidence that we Need. Indian J Dermatol
Venereol Leprol 2017;83:281-84.DOI: 10.4103/ijdvl. IJDVL_224_17.

43. Pielop, J.; Rosen, T. Penile dermatophytosis. J Am Acad Dermatol 2001;44:864-67. DOI: org/10.1067/mjd.2011.112923

44. Bardazzi, F., Marzaduri, S., Landi, C., D’Antuono, A. Microsporum infection of Penis. Genitourin Med 1997;73:579-81.

45. Dekio, S., Jidoi, J. Tinea of glans penis. Dermatolgica 1989;178:112-14.

46. Palleschi, G.M., Guadagni, R., Difonzo, E., Panconesi, E. Tinea of penis: a rareoccurrence. Int J Dermatol 1986;25:52-3.

47. Dogra, S., Uprety, S. The menace of chronic and recurrent dermatophytosis in India: Is the problem deeper than we perceive? Indian. Dermatol, Online. J.2016;7(2):73-6. DOI: 10.4103/2229-5178.178100

48. Wagenknecht, D., Simon, J.C., Treudler, R. Pubogenital tinea after intimate shaving with isolation of Trichophyton benhamiae. J. Dtsch. Dermatol. Ges. 2018;16:596-98. DOI: 10.1111/ddg. 13500

How to cite this article: Thakur R., Kushwaha P., Kalsi A., Singh P. Tinea genitalis in a rural tertiary care hospital of Western U.P. India. Indian J Clin Exp Dermatol 2018;4(4):266-73. 